\title{
The Relationship Between Physical \\ Activity and Diet Quality in Preschool \\ Children with Obesity
}

by

Alysia Vega

A thesis submitted to the Faculty of the University of Delaware in partial

fulfillment of the requirements for the degree of Honors Bachelor of Science in Dietetics with Distinction

May 2019

(C) 2019 Alysia Vega

All Rights Reserved 


\section{The Relationship Between Physical Activity and Diet Quality in Preschool \\ Children with Obesity}

by

Alysia Vega

Approved:

Shannon Robson, Ph.D.

Professor in charge of thesis on behalf of the Advisory Committee

Approved:

Jillian Trabulsi, Ph.D.

Committee member from the Department of Behavioral Health and Nutrition

Approved:

Elizabeth Orsega-Smith, Ph.D.

Committee member from the Board of Senior Thesis Readers

Approved:

Earl Lee II, Ph.D.

Deputy Faculty Director, University Honors Program 


\section{ACKNOWLEDGMENTS}

First, I would like to thank my thesis advisor, Dr. Shannon Robson. Writing my thesis would not have been possible without her consistent support and patience over the last year. Dr. Robson has shaped my undergraduate experience at the University of Delaware, from being my mentor for the last three years, to helping me meet the deadline to present at a national conference. I am truly thankful for her support and guidance and I can honestly say I would not be the student I am today without her. Thank you for believing in me and always providing a helping hand, regardless of the time of day.

I would also like to thank Dr. Jillian Trabulsi and Dr. Beth Orsega-Smith for

serving on my thesis committee and Dr. Lori Stark at Cincinnati Children's Hospital Medical Center for allowing me to use these data from the LAUNCH study.

Lastly, I would like to thank my parents for their constant support throughout this process and my entire undergraduate career. 


\section{TABLE OF CONTENTS}

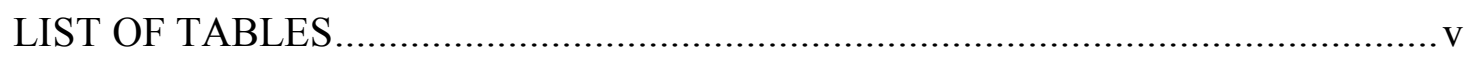

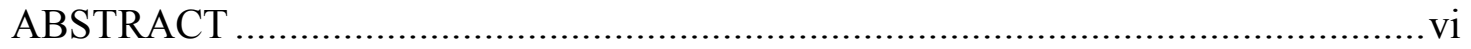

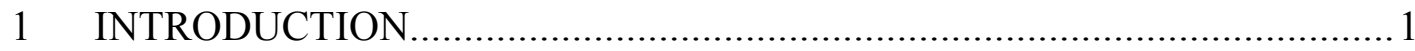

2 REVIEW OF LITERATURE ............................................................ 3

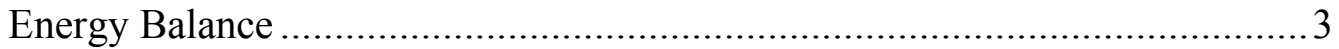

Coaction of Energy Balance Behaviors.................................................... 7

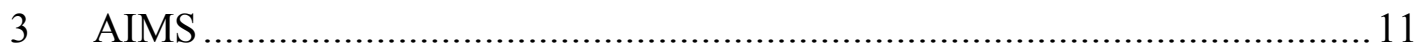

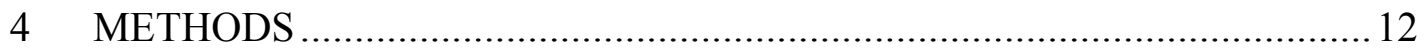

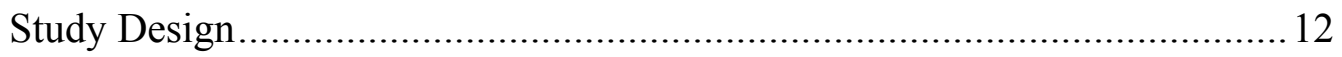

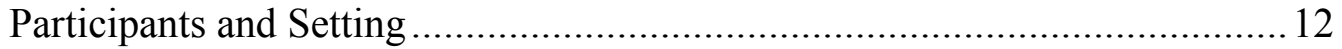

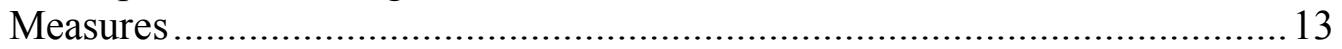

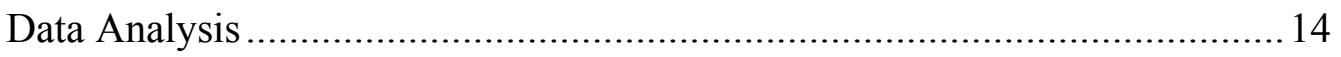

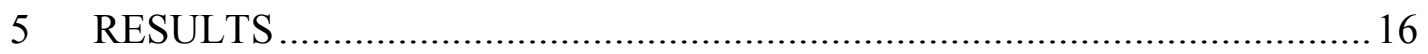

Normality/Distributions of Variables...................................................... 16

Demographic and Anthropometric Characteristics................................... 16

Energy Balance Behaviors................................................................. 16

Primary Analysis.............................................................................. 17

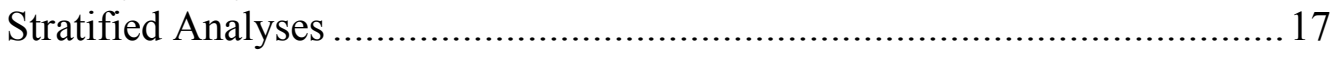

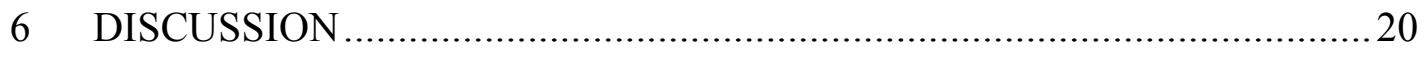

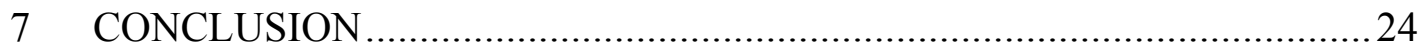

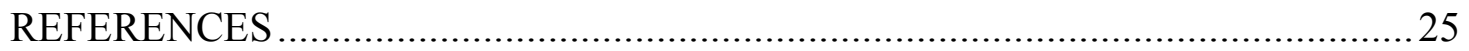




\section{LIST OF TABLES}

Table A.1. Demographic Characteristics

Table A.2. Anthropometrics

Table A.3. Physical Activity Engagement

Table A.4. Average Energy Intake and Healthy Eating Index Scores for Preschool Children

Table A.5. Correlations between HEI-2010 and MVPA in Total Sample 


\begin{abstract}
Coaction is the extent to which engaging in one behavior increases the odds of engaging in a second behavior. In adolescents and adults, engagement in physical activity has been positively associated with diet quality indicators, with the strength of the correlation often increasing with age - such that those more engaged in physical activity have a higher diet quality. The preschool years are a critical time when physical activity and eating habits are being established, but it is unknown if coaction between physical activity and diet quality exists. The purpose of this study was to examine the relationship between physical activity and diet quality in preschool children (2-5 years old) with obesity.

A secondary analysis of 148 preschool children with obesity with at least two, 24- hour dietary recalls and three days ( $\geq 5$ hours/day) of physical activity data, measured by an accelerometer, was conducted. Dietary recalls were analyzed using Nutrition Data Systems for Research (NDS-R) software and diet quality was assessed using the Healthy Eating Index (HEI-2010) total score and subcomponent scores. Physical activity was assessed by engagement in average daily minutes of moderateto-vigorous physical activity (MVPA). Pearson correlations were used to examine the relationship between diet quality and MVPA for the overall sample. Stratified analyses were also conducted by age, sex, family income, minutes of MVPA and body mass index (BMI) percentile subgroups.
\end{abstract}


On average children had a HEI-2010 total score of $56.8 \pm 10.7$ and participated in $92.6 \pm 31.7$ minutes of MVPA per day. There was no significant relationship between MVPA and HEI-2010 total score $(r=0.049, \mathrm{p}=0.56)$ or any of the HEI subcomponent scores. Among stratified analyses, in children two through three years old, average MVPA was significantly correlated with the HEI-2010 subcomponent greens and beans $(\mathrm{r}=0.428, \mathrm{p}=0.011)$. In children five years old, average MVPA was significantly correlated with total protein $(r=0.271, p=0.038)$. Similarly, for children living in households with an annual income $\geq \$ 75,000$, MVPA was significantly correlated with the HEI-2010 subcomponent score for total fruit $(\mathrm{r}=$ $0.281, \mathrm{p}=0.018$ ). Additionally, among children with $<60$ minutes of MVPA per day, the average MVPA was significantly correlated with the HEI-2010 subcomponent for sodium $(r=-0.376, p=0.044)$. Lastly, in children with a BMI $<98^{\text {th }}$ percentile, average MVPA was significantly correlated with the HEI-2010 subcomponent sodium $(\mathrm{r}=-0.333, \mathrm{p}=0.027)$

In preschool children with obesity, coaction between MVPA and diet quality does not appear to be present. Future research should examine if weight status impacts coaction between physical activity and diet quality, and where in the life course coaction between these two behaviors may be established. 


\section{Chapter 1}

\section{INTRODUCTION}

The prevalence of childhood obesity remains higher than previous years. Based on the most current data from the 2015-2016 National Health and Nutrition Examination Survey (NHANES), the national obesity rate of children 2-19 years-old is $18.5 \%{ }^{1}$ The prevalence of obesity for children $2-5$ years-old is $13.9 \%$, which is more than double the obesity rate of 5\% from the 1976-1980 NHANES survey. ${ }^{1}$ In children, weight status is defined using body mass index (BMI) percentile based upon a child's age and sex. Children who have a BMI for age and sex $\geq 85^{\text {th }}$ percentile but $<95^{\text {th }}$ percentile are classified as overweight. A child with a BMI for age and sex $\geq 95^{\text {th }}$ percentile is classified as obese. ${ }^{2}$

Starting at a young age, children with obesity suffer from a greater number of health and psychosocial consequences as compared to their healthy weight counterparts. Health consequences associated with obesity include high blood pressure, ${ }^{3}$ high cholesterol, ${ }^{4}$ impaired glucose tolerance, insulin resistance, type 2 diabetes, ${ }^{5}$ breathing problems such as asthma ${ }^{6}$ and sleep apnea, ${ }^{7}$ joint problems and musculoskeletal discomfort, ${ }^{8}$ fatty liver disease, ${ }^{9}$ gallstones,,${ }^{10}$ and gastro-esophageal reflux. ${ }^{11}$ While many of these comorbid conditions are diagnosed in adolescence or adulthood, risk factors for these health consequences are often seen in young children with obesity. Aside from these health risks, children with obesity may experience physiological, social, and behavioral consequences such as problems related to body image, self-esteem, social isolation, depression, and a reduced quality of life. ${ }^{12}$ Furthermore, children with obesity are more likely to have obesity as an adult. ${ }^{13}$ 
Overweight and obesity are a result of energy imbalance ${ }^{14}$ which is influenced by eating and physical activity behaviors. Coaction is the extent to which taking action on one behavior increases the odds of taking action on a second behavior. ${ }^{15}$ In this study, the coaction being studied is that between physical activity and diet quality in preschool-aged children with obesity. 


\section{Chapter 2}

\section{REVIEW OF LITERATURE}

\section{Energy Balance}

Energy balance is the relationship between energy (calories) intake consumed through eating and drinking and energy (calories) expenditure which includes the

thermic effect of food, basal metabolic rate, and physical activity. ${ }^{16}$ To reduce obesity and/or achieve a healthy weight, energy intake and energy expenditure must be modified. To reduce weight in children, energy expenditure needs to exceed the energy intake, above and beyond what is needed for growth. Weight maintenance occurs when energy intake is equal to energy expenditure. Alternatively, when energy intake exceeds what is needed for growth and energy expenditure, weight gain occurs.

Energy intake and energy expenditure are influenced by many biological, environmental and social factors. Biological factors can include hormones, genetic diseases such as Prader Willi syndrome, or early nutritional programming, such as how a child's in utero environment affects his or her weight status after birth.

Environmental factors are influences that occur across the home, school, built, and marketing/media environments. Lastly, social factors are conditions in the environment where people are born, live, learn, work, play, worship, and age that affect a wide range of health, functioning and quality-of life outcomes and risks. ${ }^{17}$

Two of the largest modifiable contributors to energy balance are dietary intake and physical activity. In children, dietary intake recommendations vary depending on age, sex and physical activity level. Recommendations from the 2015 Dietary 
Guidelines for Americans for energy intake in children aged 2-5 ranges from 1,000 to 1,600 calories per day, depending on age, sex and level of physical activity. ${ }^{18}$ Additional recommendations for energy intake from other professional organizations such as the American Heart Association also exist. The American Heart Association recommends between 1,000 and 1,400 calories per day for preschoolers who have low levels of physical activity. ${ }^{19}$ Despite these recommendations, population surveillance data such as, the National Health and Nutrition Examination Survey (NHANES) have shown children 2-3 years-old consume an average of $1,471 \mathrm{kcal}$ on a daily basis and that children 4-8 years-old consume 1,802 kcal daily (based upon 2005-2006 data), which are greater than the amounts recommended. ${ }^{19,20}$

In addition to energy intake, there are additional dietary recommendations based upon food groups to promote the appropriate intake of macronutrients and micronutrients. For preschool children, recommendations include daily consumption of 1-1.5 cups of fruit, 1-1.5 cups of vegetables, 2-4 ounces of protein, 3-5 ounces of grains, and 2 cups of dairy, depending on their age and sex. ${ }^{21}$ Despite recommendations, data from the Centers for Disease Control and Prevention found that among children 2-18 years old, $60 \%$ of children did not eat enough fruit to meet daily recommendations and $93 \%$ of children did not eat enough vegetables between 2007-2010. ${ }^{22}$ In the United States, studies consistently show that children's intake of fruits and vegetables are low. For example, Pabayo and colleagues ${ }^{23}$ found that among children 4-5 years old, only $29.6 \%$ of children were meeting the fruit and vegetable recommendations and the majority $(79.5 \%)$ of children were consuming more energydense, nutrient-poor foods like French fries, cookies and candy at least once per week. Overall, data trends show that children are consuming more energy-dense, nutrient 
poor foods instead of nutrient-dense foods like fruits and vegetables. ${ }^{23}$ Consumption of these higher energy-dense foods can contribute to energy intake.

In addition to individualized recommendations for energy intake and amounts from specific food groups, the overall quality of the diet is also considered. While there is no standardized measure for assessing diet quality, the Healthy Eating Index (HEI) is commonly used. The HEI is a measure of diet quality that is used to assess how well a person's dietary intake adheres to the Dietary Guidelines for Americans ${ }^{20}$ and can be used to monitor changes in dietary patterns over time. The Dietary Guidelines for Americans were created for nutrition and health professionals to help individuals (ages 2 and older) and their families consume a healthy, nutritionally adequate diet. ${ }^{24}$ The HEI is typically updated every five years with the Dietary Guidelines for Americans. The HEI-2010 total score was derived from 12 components, including 9 adequacy components (total fruit, whole fruit, total vegetables, greens and beans, whole grains, dairy, total protein foods, seafood and plant proteins, and fatty acids and three moderation components (refined grains, sodium, and empty calories). Higher scores for each component represent better diet quality with moderation components being reversed scored. The HEI-2010 total score ranges from 0 to 100 , with a higher total score representative of greater adherence to the Dietary Guidelines for Americans. According to the Center for Nutrition Policy and Promotion (CNPP), an HEI score less than or equal to 50 indicates a poor diet while scores between 51 and 80 indicates a need to improve the diet and a score greater than 80 indicates a healthy diet. ${ }^{25}$ Similar to the trends seen with child food group consumption data, data from 2007-2008 found the average HEI total score in children 2-17 years old was 49.8 , indicating poor diet quality. ${ }^{26}$ Recently the HEI- 
2015 guidelines were released; however, the HEI-2010 scoring index will be used given the association with the time frame of when data were collected. ${ }^{27}$

On the energy expenditure side of the energy balance equation physical and sedentary activity are key modifiable behaviors. The 2008 Physical Activity Guidelines ${ }^{28}$ recommended 60 minutes of moderate to vigorous physical activity (MVPA) per day for all children despite the literature supporting this recommendation was primarily based off children six years and older. The new 2018 Physical Activity Guidelines $^{29}$ released have further noted that children 3-5 years old should be physically active throughout the day to enhance their growth and development, with a reasonable target being three hours per day of activity of all intensities, including low, moderate and high. ${ }^{29}$ MVPA includes activities that get an individual moving fast enough or strenuously enough to burn three to six times as much energy as one would burn when sitting quietly, or exercises that are 3-6 metabolic equivalents (METs). ${ }^{30}$ Vigorous physical activity requires a large amount of effort and causes rapid breathing and substantial increase in heart rates, and burns more than six METs. ${ }^{31} \mathrm{~A}$ metaanalysis $^{32}$ of 29 different studies found that children aged 3-5 years are not meeting physical activity guidelines, with children only engaging in an average of 42.8 minutes of MVPA per day. Despite this finding, data from the original study ${ }^{33}$ used for this secondary analysis found that preschool children with obesity are meeting the daily recommendation of at least 60 minutes per day for MVPA.

To date, the majority of research has highlighted the need for improved dietary intake and physical activity behaviors in preschool-aged children. Given diet and physical activity are the two key modifiable behaviors associated with energy balance, 
modifying these behaviors can promote a healthy weight in children. Furthermore these behaviors may be practiced together to improve health outcomes.

\section{Coaction of Energy Balance Behaviors}

Good health, including a healthy weight, is often an outcome associated with engagement in more than one health behavior. For example, a healthy weight is often the result of eating healthfully and being physically activity. Given the fact that multiple behaviors can contribute to a healthy weight, it may be important to understand coaction, and if coaction occurs between dietary and physical activity behaviors. Coaction is the extent to which taking action on one behavior increases the odds of taking action on a second behavior. ${ }^{15}$

Coaction between physical activity and dietary intake has been examined more commomly in adults and adolescents as compared to younger pediatric populations. In the following, findings from the literature related to the coaction of physical activity and dietary intake will be reviewed.

In young adults, it has been seen that engagement in physical activity is related to fruit and vegetable intake. Specifically, a longitudinal study of 6,244 young adults (18-22 years old) collected information on physical activity frequency and consumption of fruit and vegetable frequency through interviews via a computerassisted personal interview. ${ }^{34}$ The study showed that physical activity frequency $(<5$ days/week vs. $\geq 5$ days per week) was linearly associated with fruit and vegetable intake (0 times/week, 1-3 times/week, 4-6 times/week, 1 time/day, 2 times/day, 3 times/day and $\geq 4$ times/day) indicating that individuals who were newly engaging in physical activity or who were continuing to engage in physical activity also had 
greater fruit and vegetable consumption over time. Matthews and colleagues ${ }^{35}$ investigated the relationship between leisure time physical activity (LTPA) and dietary variables in 425 males and 494 females who were part of a larger study (Worcester Area Trial for Counseling in Hyperlipidemia). The results showed that participants who participated in LTPA for at least 30 minutes per week consumed fewer servings per week of meats, fried foods, sweets and higher fat (2-4\%) milk, and more servings of fruits, vegetables, and low fat dairy products $(0-1 \%$ milk) than did inactive subjects. ${ }^{35}$ Similarly, when looking at diet quality in adults, LTPA was related to a diet lower in fat and cholesterol and was higher in micronutrients and fiber. ${ }^{35}$ Taken together, these studies show that when adults were more active they consumed a diet that was healthier (i.e., higher diet quality).

In adolescents, coaction between physical activity and dietary intake has also shown that engagement in physical activity is associated with a healthier dietary intake, with the strength of this correlation increasing with age. ${ }^{36}$ In a nationally representative sample of adolescents $(n=11,631)$ who responded to a physical activity survey, 2,652 were classified as high active ( $\geq 6$ days of hard and $\geq 6$ days of light exercise) and 1,641 were classified as low active ( $\leq 2$ days of light exercise and no days of hard exercise). ${ }^{37}$ Adolescents completed a questionnaire that assessed many health factors including dietary practices (e.g., fruit and vegetable consumption). Results showed that students who ate no fruit per day were more likely to be low active as compared to those who ate one or more servings of fruit per day. ${ }^{37}$ Similarly, the students who ate no vegetables per day were almost twice as a likely to be low active as compared to students who ate one or more servings of vegetables per day. ${ }^{37}$ In another study of 822 adolescents, from a large, diverse suburban high school in 
northeast Florida, Delisle and colleagues ${ }^{38}$ found that adolescents who engaged in high levels of vigorous physical activity were significantly $(\mathrm{p}<0.001)$ more likely to eat healthy carbohydrates and healthy fats as compared to adolescents who had low levels of vigorous physical activity. A similar pattern was shown for moderate level physical activity and consumption of healthy carbohydrates and healthy fats as well. ${ }^{38}$ These studies in adolescents indicate that similar to adults, coaction between physical activity and healthy dietary intake has been observed..

Although the direct relationship between physical activity and diet quality in younger children has been understudied, there are data from studies that indicate a relationship between the two health behaviors. One study ${ }^{39}$ independently examined physical activity and diet quality in relation to obesity in children 6-17 years-old, but did not specifically assess the relationship of physical activity and diet quality. Findings showed that children who consumed a healthy diet (defined as HEI-2010 score at or above the $60^{\text {th }}$ percentile) and were considered to be physically active (defined as engaging in at least 60 minutes of MVPA per day) reported an HEI-2010 total score of 53 and participated in 110 minutes of MVPA per day. ${ }^{39}$ In contrast, the children who consumed an unhealthy diet and were physically inactive had an HEI2010 total score of 36 and participated in only 27 minutes of MVPA per day. ${ }^{39}$ Furthermore, children with a higher HEI score and who were physically active were at a lower risk for overweight and obesity. ${ }^{39}$ Similarly, a study was done among a sample of 2,287 Greek preschool children between the ages of 2-5 years old to assess their diet quality and the factors that affect influence diet quality. The mean HEI total score was 59; however, the study also found that the HEI total scores were significantly higher in children participating in MVPA for at least three hours/week (HEI total 
score: $60.4 \pm 7.3)$ as compared to children participating in MVPA for less than three hours/week (HEI total score: $58.5 \pm 8.2) .^{40}$

While these data from adults and adolescent populations consistently demonstrate coaction between physical activity and diet quality, less is known about the coaction of physical activity and dietary intake behaviors in younger children, particularly preschool-aged children. Understanding coaction, and if it occurs during the preschool years is important because this is a time period when eating behaviors and physical activity behaviors are being developed. The development of healthy eating and physical activity behaviors at a young age can promote a healthy weight across the life course. In contrast, a child who develops unhealthy eating and sedentary activity habits during the preschool years may be susceptible to negative health outcomes such as overweight or obesity. 


\title{
Chapter 3
}

\begin{abstract}
AIMS
The primary goal of this research was to evaluate if coaction, the extent to which taking action on one behavior increases the chances of taking action on a second behavior, occured between physical activity and diet quality in preschool-aged children with obesity.
\end{abstract}

Specific Aim: To determine the relationship between minutes of moderate-tovigorous physical activity (MVPA) and diet quality (HEI-2010 total score) in preschool children (2-5 years-old) with obesity.

Hypothesis: MVPA will be significantly correlated with HEI-2010 total score and children with more minutes of MVPA will have a higher HEI-2010 total score. 


\section{Chapter 4}

\section{METHODS}

\section{Study Design}

A secondary data analysis was conducted using baseline data from a randomized controlled trial that examined whether a skills-based behavioral family clinic and home-based intervention (LAUNCH) or motivational interviewing was superior to the standard of care among preschool children (2-5 years-old) with obesity. ${ }^{41,42}$

\section{Participants and Setting}

The original study $(n=151)$ was conducted from March 2012 to June 2015. ${ }^{35,36}$ Full participant and setting information has been reported elsewhere. ${ }^{35,36}$ In brief, children were required to have a BMI $\geq 95^{\text {th }}$ percentile, but no more than $100 \%$ above the median BMI, be 2-5 years-old, have medical clearance from their pediatrician, be an active patient with anthropometric measurements within the previous year, speak English and live within 50 miles of the medical center where the study was being conducted. ${ }^{33,34}$ For this secondary analysis, additional eligibility criteria included having at least two, 24-hour dietary recalls and complete physical activity data defined as having worn the ActiGraph, an accelerometer that captures physical activity, for a minimum of five hours per day on at least three days from the baseline assessment. Given these criteria the sample for this study was 148 preschoolaged children. The original study was approved by the Institutional Review Board at Cincinnati Children's Hospital's Medical Center. 


\section{Measures}

Demographics. Caregivers completed a questionnaire regarding child and caregiver date of birth, race, ethnicity, and sex, and caregiver education, occupation, marital status, and family income. Caregivers' education and occupation were used to calculate a family's socioeconomic status using the Hollingshead 4-factor Index of Socioeconomic Status where scores range from 8 to 66 with higher numbers indicating higher socioeconomic status. ${ }^{43}$

Dietary Intake. Child dietary intake was assessed using three random 24-hour recalls (two weekdays, one weekend day) using the multi-pass method. The first recall was completed in person during the baseline assessment with the two additional recalls completed over the phone. All dietary recalls were completed by a trained research dietitian. Parents were provided with 2-dimensional food portion size models to assist with the recalls. Dietary recalls were analyzed using Nutrition Data Systems for Research (NDS-R) software (versions 2012-2016, Nutrition Coordinating Center, University of Minnesota) and diet quality was determined using the Healthy Eating Index (HEI-2010). ${ }^{44}$ Data from dietary recalls were averaged across the three days to obtain an average intake per day. The HEI-2010 evaluates diet quality based upon adherence to the 2010 Dietary Guidelines for Americans. ${ }^{45}$ The HEI-2010 is derived from 12 components, including nine adequacy components (total fruit, whole fruit, total vegetables, greens and beans, whole grains, dairy, total protein foods, seafood and plant proteins, and fatty acids) and three moderation components (refined grains, 
sodium, and empty calories) ${ }^{44}$ Higher scores for each component represent better diet quality with moderation components being reversed scored. HEI-2010 total score ranges from 0 to 100, with 100 indicating perfect adherence to the Dietary Guidelines. Physical Activity. Child physical activity was monitored using an ActiGraph accelerometer (Model GT3X+), validated and calibrated for use among preschool children. ${ }^{46}$ The ActiGraph accelerometer was a small device worn around the waist of the child. Accelerometers measure acceleration (counts) in real time and detect movement along three different orthogonal planes. Physical activity was measured as average minutes of MVPA per day. ${ }^{47}$

Anthropometrics. Child height (Holtain stadiometer) and weight (Scaletronix, Wheaton, IL) were measured using standard procedures. Height and weight were used to calculate child BMI percentile and BMI z-score.

\section{Data Analysis}

Data were analyzed for skewness, kurtosis, and extreme outliers using histograms and stem and leaf plots. As needed, transformations or non-parametric tests were used if assumptions were violated. Means and frequencies were used to describe continuous (e.g. age, weight) and categorical (e.g. sex, race) data, respectively. To assess the specific aim, Pearson correlations were used to examine the relationship between physical activity and diet quality for the overall sample. Pearson correlations were also used for stratified analyses within demographic subgroups (e.g. age, sex, 
family income, minutes of MVPA, BMI percentile). Statistical analysis was completed using SPSS version 23.0 (IBM Corporation) at a significance level of alpha $<0.05$. 


\section{Chapter 5}

\section{RESULTS}

\section{Normality/Distributions of Variables}

All variables were normally distributed so no transformations were made. There were no extreme outliers. All data were included in analyses.

\section{Demographic and Anthropometric Characteristics}

Participants $(n=148)$ in the study were $55.2 \pm 11.2$ months old and were $42.6 \%$ male and $57.4 \%$ female. The majority $(75.7 \%)$ of participants identified their race to be White or Caucasian and $6.1 \%$ of the sample identified as Hispanic or Latino. The annual household income $(\mathrm{n}=147)$ reported by $51.7 \%$ of parents ranged between $\$ 50,000-\$ 99,000$ and the average Hollingshead Score was $43.0 \pm 11.7$ indicating that the members hold a relatively high status in society since the score range is 8 to 66 , with higher numbers indicating a higher status in society. ${ }^{43}$ For additional demographic details see Table A.1.

Children had a mean height of $111.2 \mathrm{~cm} \pm 8.2 \mathrm{~cm}$ and mean weight of $26.1 \mathrm{~kg}$ $\pm 5.6 \mathrm{~kg}$. Average BMI percentile was $98.6 \pm 1.3$ and BMI z-score was $2.4 \pm 0.6$, indicating children had obesity. See Table A.2.

\section{Energy Balance Behaviors}

As shown in Table A.3 children participated in an average of 92.6 minutes \pm 31.7 minutes of MVPA. Over the course of a day, children spent $12.0 \% \pm 3.8 \%$ of their time engaging in MVPA. Children consumed an average of 1,401 $\pm 316 \mathrm{kcal} /$ day and had an HEI-2010 total score of $56.8 \pm 10.7$ (HEI-2010 range 0-100). HEI-2010 
subcomponent scores are shown in Table A.4. The maximum subcomponent score was not achieved for any category.

\section{Primary Analysis}

The correlation between minutes of MVPA and HEI-2010 total score $(r=$ $0.049, p=0.558)$ was extremely weak and not significiant. Furthermore, there was no statistically significant $(p>0.05)$ correlation between minutes of MVPA and any of the HEI subcomponent scores. While not significant, average MVPA had a negative correlation with the following HEI-2010 subcomponent scores: total vegetables, sodium and refined grains. MVPA was also not significantly correlated with total energy intake $(r=0.129, p=0.119)$. See Table A.5.

\section{Stratified Analyses}

Stratified analyses examined the relationship between MVPA and HEI total score within specific demographic variables including: age (1-3 years vs. $4-5$ years and 2-4 years vs. 5 years); sex (male vs. female), and annual household income $(<\$ 75,000$ vs. $\geq \$ 75,000)$. Data were also further investigated based upon the recommendation for children to engage in at least 60 minutes of MVPA per day $(<60$ $\mathrm{min} /$ day vs. $\geq 60 \mathrm{~min} /$ day) and BMI percentile ( $<98$ th percentile vs $\geq 98$ th percentile).

Age. When children were examined by age, stratified in groups of two to three years versus four to five years, in children two to three years old $(n=34)$ average MVPA was not significantly correlated with HEI-2010 total score $(r=-0.014, p=$ 0.938), but was significantly correlated with the subcomponent greens and beans $(\mathrm{r}=$ $0.428, p=0.011)$. No other HEI subcomponent scores were significant within the two 
to three year old subgroup. In children 4-5 $(n=114)$ average MVPA was not significantly correlated with HEI-2010 total score $(r=0.099, p=0.293)$ or any of the subcomponent scores. Similarly, when the relationship between MVPA and HEI-2010 total score was examined in children two to four years old $(n=89)$ and children five years old $(\mathrm{n}=59)$, no significant correlation was found. MVPA was significantly correlated with total protein $(\mathrm{r}=0.271, \mathrm{p}=0.038)$ in children five years old.

Sex. In males $(\mathrm{n}=63)$ MVPA was not significantly correlated with HEI-2010 total score $(\mathrm{r}=0.140, \mathrm{p}=0.275)$ or any of the HEI-2010 subcomponent scores. In females $(\mathrm{n}=85)$ MVPA was not significantly correlated with HEI-2010 total score ( $\mathrm{r}$ $=-0.074, \mathrm{p}=0.501)$ or any of the HEI-2010 subcomponent scores.

Family Income. In children with an annual family income $<\$ 75,000(\mathrm{n}=77)$, MVPA was not significantly correlated with HEI-2010 total score $(r=-0.034, \mathrm{p}=$ 0.770) or any of the HEI-2010 subcomponent scores. In children with an annual family income $\geq \$ 75,000(\mathrm{n}=70)$ MVPA was not significantly correlated with HEI2010 total score $(r=0.152, p=0.210)$. MVPA was significantly correlated with the subcomponent total fruit $(\mathrm{r}=0.281, \mathrm{p}=0.018)$ in children with a family income $\geq \$ 75,000$. MVPA was not significantly correlated with any other HEI-2010 subcomponent scores in children with an annual family income $\geq \$ 75,000$.

Minutes of MVPA. In children with $<60$ minutes of MVPA $(\mathrm{n}=29)$ MVPA was not significantly correlated with HEI-2010 total score $(r=-0.013, p=0.946)$. In these children, MVPA was significantly correlated with the subcomponent sodium $(\mathrm{r}=$ $-0.376, \mathrm{p}=0.044)$. MVPA was not significantly correlated with any other HEI-2010 subcomponent scores in children with $<60$ minutes of MVPA daily. In children with 
$\geq 60$ minutes of daily MVPA $(n=119)$, MVPA was not significantly correlated with HEI-2010 total score $(\mathrm{r}=0.098, \mathrm{p}=0.290)$ or any HEI subcomponent scores.

BMI Percentile. In children with a BMI percentile $<98(\mathrm{n}=44)$, MVPA was not significantly correlated with HEI-2010 total score $(r=-0.191, p=0.215)$. MVPA was significantly correlated with the subcomponent score sodium $(\mathrm{r}=-0.333, \mathrm{p}=$ 0.027). MVPA was not significantly correlated with any other HEI-2010 subcomponent score in children with a BMI percentile $<98$. In children with a BMI percentile $\geq 98(\mathrm{n}=104)$ average MVPA was not significantly correlated with HEI2010 total score $(r=0.148, p=0.135)$ or any of the HEI-2010 subcomponent scores. 


\section{Chapter 6}

\section{DISCUSSION}

The primary aim of this study was to look at coaction, the extent to which taking action on one behavior increases the chances of taking action on a second behavior, between physical activity and diet quality in preschool-aged children with obesity. ${ }^{15}$

The overall total HEI-2010 total score for the children was $56.8 \pm 10.7$ indicating a need to improve their diet. ${ }^{25}$ Children on average participated in 92.6 minutes \pm 31.7 minutes of MVPA, which is above the recommended 60 minutes per day. ${ }^{28}$ There was no relationship seen in the children between their minutes of MVPA per day and HEI-2010 total score; however MVPA was significantly correlated with several HEI-2010 subcomponents scores within the stratified analyses.

Despite the supporting literature in adults and adolescents, the coaction between physical activity and diet quality in preschool-aged children with obesity was not observed in this study. Given this finding, it may be important to think about where in the life course coaction between physical activity and diet quality begins to develop. Research shows that by adolescence this coaction is seen and it continues on through both young adulthood and adulthood. ${ }^{28-32}$ Therefore, the lack of findings could be due to the fact that the children being studied were too young and had not yet developed the relationship between these behaviors. Similarly, various studies suggest that parents are responsible for what young children eat. ${ }^{48,49}$ Since the child is not directly picking out his or her foods, the mechanism leading to the coaction seen in adolescents and adults may not exist yet. 
Children in this study were also already achieving high levels of physical activity ( $81.5 \%$ of children were achieving 60 or more minutes of MVPA per day), perhaps providing too little variability within the data for a significant finding. This high level of activity is contrary to data that has demonstrated preschool-aged children are not meeting physical activity recommendation $\mathrm{s}^{32}$ and given the fact that children with obesity often have lower levels of physical activity as compared to their healthy weight counterparts. ${ }^{50}$ It may be important to further examine physical activity to understand if there is a threshold in the relationship to diet quality. If a threshold were to exist, once a child is above a certain number of minutes of physical activity, diet quality may not improve regardless of the number of minutes a child were to be active.

While children in this study exceeded physical activity recommendations, diet quality was poor. Given that the children have obesity, these data suggest that dietary intake may be a larger contributor to their high weight status due to the high level of energy expenditure through physical activity. In adolescents aged 10-19 years old, data have shown that adolescents in the highest quartile of HEI-2010 had a lower risk of general obesity and central obesity as compared to adolescents in the lowest

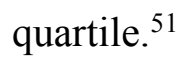

Given that all of the children had obesity, the lack of findings may be unique to this population. In a study with adults, analyses examining the association between physical activity and fruit and vegetable consumption controlled for factors such as body mass index ${ }^{34}$ indicating that weight status may play a role in the relationship between physical activity and dietary intake. This means that the weight status of the children in the study could have influenced the results, and coaction may be prevalent in preschoolers of a healthy weight, but the sample did not allow for this to be 
examined. Future research might consider looking at the relationship between physical activity and diet quality within weight status categories.

With the lack of significant findings, directionality of the relationships was further examined given one would expect with coaction that physical activity and diet quality would have a direct relationship. For total HEI-2010 score and MVPA, the relationship between physical activity and diet quality was direct, though it was a weak correlation and not statistically significant. When subgroups were examined it was suprising to not see differences, specifically within income given that consuming a diet higher in diet quality is often hypothesized to be more expensive. ${ }^{52,53}$ One metaanalysis that examined the prices of healthier versus less healthy foods across multiple countries and diet patterns included a sub-analsis of US only studies and found that healthier food-based diet patterns cost an average of $\$ 1.49$ more than less healthy eating patterns. ${ }^{52}$ When looking more closely at the income stratified analysis, in children who came from a family whose annual income was below $\$ 75,000$, the relationship between physical activity and diet quality was negative, meaning there was an inverse relationship - the opposite of what one would expect to see if coaction was occurring. In contrast, for the group of children who came from a family with an annual income $\geq \$ 75,000$, the relationship was positive, meaning there was a direct relationship between physical activity and diet quality, with diet quality increasing (or decreasing) as the minutes of MVPA increased (or decreased).

When looking at the subgroups, specifically when divided by sex, the directionality shows that the relationship between physical activity and diet quality is inverse in females but linear in males. This means that in girls as diet quality increased 
(or decreased), the minutes of MVPA decreased (or increased) but in males, as diet quality increased (or decreased), the minutes of MVPA increased (or decreased).

Despite limited findings, there are several strengths of the study, particularly the use of an objective assessment for physical activity and three, random 24-hour recalls for dietary intake which provide a good representation of usual intake. Limitations of the study include that results are not generalizable to older children or preschool-aged children who do not have obesity. The sample was also primarily Caucasion (75.7\%) and came from the same geographic region, further limiting generalizability to a broader population. Similarly, it was a cross sectional study, meaning the participants were only examined at one point in time. 


\section{Chapter 7}

\section{CONCLUSION}

In preschool-aged children with obesity, coaction between physical activity behavior and diet quality was not apparent. The majority of children (81.5\%) were meeting the recommended 60 minutes of MVPA per day, but had a diet quality that was in need of improvement based on HEI-2010 classification. Given that all of the children had obesity, this study showed that poor diet quality may be a larger contributor to energy imbalance than enery expenditure. 


\section{REFERENCES}

If you want to number your bibliographic entries, change the style of the items to $\mathrm{Bib}$ Entry - numbered.

1. Robert Wood Johnson Foundation. The State of Obesity. https://stateofobesity.org/childhood-obesity-trends/. Accessed December 4, 2018.

2. Centers for Disease Control and Prevention. Defining Childhood Obesity. https://www.cdc.gov/obesity/childhood/defining.html. Accessed December 4, 2018.

3. Rosaneli CF, Baena CP, Auler F, et al. Elevated blood pressure and obesity in childhood: a cross-sectional evaluation of 4,609 schoolchildren. Arq Bras Cardiol. 2014;103(3):238-244.

4. Korsten-Reck U, Kromeyer-Hauschild K, Korsten K, Baumstark MW, Dickhuth HH, Berg A. Frequency of secondary dyslipidemia in obese children. Vasc Health Risk Manag. 2008;4(5):1089-1094.

5. Ferrannini E, Camastra S. Relationship between impaired glucose tolerance, non-insulin-dependent diabetes mellitus and obesity. Eur J Clin Invest. 1998;28 Suppl 2:3-6; discussion 6-7.

6. Lang JE. Obesity and asthma in children: current and future therapeutic options. Paediatr Drugs. 2014;16(3):179-188.

7. Evans CA, Selvadurai H, Baur LA, Waters KA. Effects of obstructive sleep apnea and obesity on exercise function in children. Sleep. 2014;37(6):11031110.

8. Smith SM, Sumar B, Dixon KA. Musculoskeletal pain in overweight and obese children. Int J Obes (Lond). 2014;38(1):11-15.

9. Fabbrini E, Sullivan S, Klein S. Obesity and nonalcoholic fatty liver disease: biochemical, metabolic, and clinical implications. Hepatology. 2010;51(2):679-689.

10. Erlinger S. Gallstones in obesity and weight loss. Eur J Gastroenterol Hepatol. 2000;12(12):1347-1352.

11. Chang P, Friedenberg F. Obesity and GERD. Gastroenterol Clin North Am. 2014;43(1):161-173.

12. Pulgarón ER. Childhood obesity: a review of increased risk for physical and psychological comorbidities. Clin Ther. 2013;35(1):A18-32.

13. Centers for Disease Control and Prevention. Childhood Obesity Causes and Consequences. https://www.cdc.gov/obesity/childhood/causes.html. Accessed December 4, 2018.

14. Hill J WH, Peters J. Energy Balance and Obesity. PMC. 2013;126(1):126-132.

15. Johnson SS, Paiva AL, Mauriello L, Prochaska JO, Redding C, Velicer WF. Coaction in multiple behavior change interventions: consistency across 
multiple studies on weight management and obesity prevention. Health Psychol. 2014;33(5):475-480.

16. U.S. Department of Health and Human Services. Balance Food and Activity. https://www.nhlbi.nih.gov/health/educational/wecan/healthy-weightbasics/balance.htm. Accessed December 4, 2018.

17. Office of Disease Prevention and Health Promotion. Social Determinants of Health. https://www.healthypeople.gov/2020/topics-objectives/topic/socialdeterminants-of-health. Accessed December 4, 2018.

18. United States Department of Agriculture. How Much Does my Preschooler Need. https://www.choosemyplate.gov/how-much-does-my-preschooler-need. Accessed December 4, 2018.

19. American Heart Association. Dietary Recommendations for Healthy Children. https://www.heart.org/en/healthy-living/healthy-eating/eat-smart/nutritionbasics/dietary-recommendations-for-healthy-children. Published 2018. Accessed March 23, 2019.

20. United States Department of Agriculture. Dietary Guidelines for Americans 2015-2020 Eighth Edition. Accessed December 4, 2018.

21. Mayo Clinic. Nutrition for Kids: Guidelines for a Healthy Diet. https://www.mayoclinic.org/healthy-lifestyle/childrens-health/indepth/nutrition-for-kids/art-20049335. Accessed 2018, December 4.

22. Centers for Disease Control and Prevention. Children Eating More Fruit, but Fruit and Vegetable Intake Still Too Low. https://www.cdc.gov/media/releases/2014/p0805-fruits-vegetables.html. Published 2014. Updated August 5, 2014. Accessed April 9, 2019.

23. Pabayo R, Spence JC, Casey L, Storey K. Food consumption patterns in preschool children. Can J Diet Pract Res. 2012;73(2):66-71.

24. United States Department of Agriculture. Healthy Eating Index (HEI). https://www.cnpp.usda.gov/healthyeatingindex. Accessed December 4, 2018.

25. Snetselaar L. Are Americans Following US Dietary Guidelines? Check the Healthy Eating Index. https://www.elsevier.com/connect/are-americansfollowing-us-dietary-guidelines-check-the-healthy-eating-index. Published 2015. Updated March 2, 2015. Accessed April 12, 2019.

26. United States Department of Agriculture. Diet Quality of Children Age 2-17 Years as Measured by the Healthy Eating Index-2010. https://www.cnpp.usda.gov/sites/default/files/nutrition insights uploads/Insig ht52.pdf. Published 2013. Accessed December 22, 2018.

27. U.S. Department of Health and Human Services. Comparing the HEI-2015, HEI-2010 and HEI-2005. https://epi.grants.cancer.gov/hei/comparing.html. Accessed December 4, 2018.

28. U.S. Department of Health and Human Services. 2008 Physical Activity Guidelines for Americans. In.

29. U.S. Department of Health and Human Services. Physical Activity Guidelines for Americans. https://health.gov/paguidelines/second- 
edition/pdf/Physical_Activity_Guidelines 2nd edition.pdf. Accessed December 4, 2018.

30. Harvard T.H. Chan. Examples of Moderate and Vigorous Physical Activity. https://www.hsph.harvard.edu/obesity-prevention-source/moderate-andvigorous-physical-activity/. Accessed December 4, 2018.

31. World Health Organization. What is Moderate-Intensity and Vigorous Intensity Physical Activity. http://www.who.int/dietphysicalactivity/physical activity intensity/en/. Accessed December 4, 2018.

32. Bornstein DB, Beets MW, Byun W, McIver K. Accelerometer-derived physical activity levels of preschoolers: a meta-analysis. J Sci Med Sport. 2011;14(6):504-511.

33. Odar Stough C, McCullough M, Robson S, et al. Are Preschoolers Meeting the Mark? Comparing the Dietary, Activity, and Sleep Behaviors of Preschoolers with Obesity to National Recommendations. J Pediatr Psychol. 2018;43(4):452-463.

34. Jayawardene WP, Torabi MR, Lohrmann DK. Exercise in Young Adulthood with Simultaneous and Future Changes in Fruit and Vegetable Intake. J Am Coll Nutr. 2016;35(1):59-67.

35. Matthews CE, Hebert JR, Ockene IS, Saperia G, Merriam PA. Relationship between leisure-time physical activity and selected dietary variables in the Worcester Area Trial for Counseling in Hyperlipidemia. Med Sci Sports Exerc. 1997;29(9):1199-1207.

36. Maier JH, Barry R. Associations among Physical Activity, Diet, and Obesity Measures Change during Adolescence. J Nutr Metab. 2015;2015:805065.

37. Pate RR, Heath GW, Dowda M, Trost SG. Associations between physical activity and other health behaviors in a representative sample of US adolescents. Am J Public Health. 1996;86(11):1577-1581.

38. Delisle TT, Werch CE, Wong AH, Bian H, Weiler R. Relationship between frequency and intensity of physical activity and health behaviors of adolescents. $J$ Sch Health. 2010;80(3):134-140.

39. An R. Diet quality and physical activity in relation to childhood obesity. Int J Adolesc Med Health. 2017;29(2).

40. Manios Y, Kourlaba G, Kondaki K, et al. Diet quality of preschoolers in Greece based on the Healthy Eating Index: the GENESIS study. J Am Diet Assoc. 2009;109(4):616-623.

41. Stark LJ, Filigno SS, Bolling C, et al. Learning about Activity and Understanding Nutrition for Child Health (LAUNCH): Rationale, design, and implementation of a randomized clinical trial of a family-based pediatric weight management program for preschoolers. Contemp Clin Trials. 2017;52:10-19. 
42. Stark LJ, Spear Filigno S, Bolling C, et al. Clinic and Home-Based Behavioral Intervention for Obesity in Preschoolers: A Randomized Trial. J Pediatr. 2018;192:115-121.e111.

43. Hollingshead A. Four Factor Index of Social Status. In.

44. Guenther P, Casavale K, Reedy J, et al. Update of the Healthy Eating Index: HEI-2010. J Am Diet Assoc. 2013;113(4):569-580.

45. U.S. Department of Health and Human Services. Dietary Guidelines for Americans. http://health.gov/dietaryguidelines/. Published 2010. Accessed July 31, 2014.

46. Pate RR, Almeida MJ, McIver KL, Pfeiffer KA, Dowda M. Validation and calibration of an accelerometer in preschool children. Obesity (Silver Spring). 2006;14(11):2000-2006.

47. Sylvia LG, Bernstein EE, Hubbard JL, Keating L, Anderson EJ. Practical guide to measuring physical activity. J Acad Nutr Diet. 2014;114(2):199-208.

48. Jang M, Brandon D, Vorderstrasse A. Relationships Among Parental Psychological Distress, Parental Feeding Practices, Child Diet, and Child BMI. Nurs Res. 2019.

49. Berhane HY, Ekström EC, Jirström M, et al. What Influences Urban Mothers' Decisions on What to Feed Their Children Aged Under Five-The Case of Addis Ababa, Ethiopia. Nutrients. 2018;10(9).

50. Hills AP, Andersen LB, Byrne NM. Physical activity and obesity in children. Br J Sports Med. 2011;45(11):866-870.

51. Mohseni-Takalloo S, Hosseini-Esfahani F, Mirmiran P, Azizi F. Associations of Pre-Defined Dietary Patterns with Obesity Associated Phenotypes in Tehranian Adolescents. Nutrients. 2016;8(8).

52. Rao M, Afshin A, Singh G, Mozaffarian D. Do healthier foods and diet patterns cost more than less healthy options? A systematic review and metaanalysis. BMJ Open. 2013;3(12):e004277.

53. Jones NR, Conklin AI, Suhrcke M, Monsivais P. The growing price gap between more and less healthy foods: analysis of a novel longitudinal UK dataset. PLoS One. 2014;9(10):e109343. 


\section{Appendix A}

Table A.1. Demographic Characteristics

\begin{tabular}{|l|l|}
\hline & \multicolumn{1}{|c|}{$\mathrm{n}=148$} \\
\hline Age (in months) & $55.2 \pm 11.2$ \\
\hline Sex, n(\%) & $63(42.6 \%)$ \\
Male & $85(57.4 \%)$ \\
Female & $112(75.7 \%)$ \\
\hline Race, n(\%) & $14(9.5 \%)$ \\
White or Caucasian & 0 \\
Black or African American & 0 \\
Asian & 0 \\
American Indian or Alaskan & 0 \\
Native & 0 \\
Native Hawaiian or Pacific & $3(2 \%)$ \\
Islander & 0 \\
Other & $19(12.8 \%)$ \\
Unknown & \\
More than one race & $9(6.1 \%)$ \\
\hline Ethnicity, n(\%) & $139(93.9 \%)$ \\
Hispanic and Latino & \\
Not Hispanic and Latino & \\
\hline Family Income n=147, & $7(4.8 \%)$ \\
n(\%) & $3(2 \%)$ \\
Under \$10,000 & $6(4.1 \%)$ \\
\$10,000-\$19,999 & $11(7.5 \%)$ \\
\$20,000-\$29,999 & $11(7.5 \%)$ \\
\$30,000-\$39,999 & $39(26.5 \%)$ \\
\$40,000-\$49,999 & $37(25.2 \%)$ \\
\$50,000-\$74,999 & $19(12.9 \%)$ \\
\$75,000-\$99,999 & $5(3.4 \%)$ \\
\$100,000-\$124,999 & $3(2 \%)$ \\
\$125,000-\$149,999 & 0 \\
\$150,000-\$174,999 & $6(4.1 \%)$ \\
\$175,000-\$199,999 & $43.0 \pm 11.7$ \\
\$200,000 or more & \\
\hline Hollingshead Score & 0 \\
\hline
\end{tabular}


Table A.2. Anthropometrics

\begin{tabular}{|l|l|}
\hline & $\mathrm{n}=148$ \\
\hline Height $(\mathrm{cm})$ & $111.2 \pm 8.2$ \\
\hline Weight $(\mathrm{kg})$ & $26.1 \pm 5.6$ \\
\hline BMI & $20.9 \pm 2.5$ \\
\hline BMI z-score & $2.4 \pm 0.6$ \\
\hline BMI Percentile & $98.6 \pm 1.3$ \\
\hline
\end{tabular}


Table A.3. Average Minutes and Percent Time of Moderate-to-Vigorous Physical Activity

\begin{tabular}{|l|c|}
\hline & $\mathrm{n}=148$ \\
\hline Average MVPA (in minutes) & $92.6 \pm 31.7$ \\
\hline Average \%Time in MVPA & $.12 \pm .04$ \\
\hline
\end{tabular}

MVPA, Moderate-to-vigorous physical activity defined as exercises that are 3-6 metabolic equivalents (METs) ${ }^{30}$ 
Table A.4. Average Energy Intake and Healthy Eating Index Scores for Preschool Children

\begin{tabular}{|l|c|c|}
\hline & Score Range & $\mathrm{n}=148$ \\
\hline Total Energy Intake & $\mathrm{N} / \mathrm{A}$ & $1401 \pm 316$ \\
\hline Total HEI Score & $0-100$ & $56.8 \pm 10.7$ \\
\hline Total Vegetables & $0-5$ & $3.0 \pm 1.5$ \\
\hline Greens and Beans & $0-5$ & $0.6 \pm 1.3$ \\
\hline Total Fruit & $0-5$ & $3.3 \pm 1.7$ \\
\hline Whole Fruit & $0-5$ & $3.5 \pm 1.8$ \\
\hline Whole Grains & $0-10$ & $3.6 \pm 3$ \\
\hline Dairy & $0-10$ & $8.9 \pm 1.9$ \\
\hline Total Protein & $0-5$ & $3.9 \pm 1.3$ \\
\hline $\begin{array}{l}\text { Seafood and Plant } \\
\text { Proteins }\end{array}$ & $0-5$ & $1.8 \pm 1.9$ \\
\hline Fatty Acids & $0-10$ & $3.6 \pm 3.0$ \\
\hline Sodium & $0-10$ & $4.3 \pm 2.7$ \\
\hline Refined Grains & $0-10$ & $6.0 \pm 3.2$ \\
\hline Discretionary Calories & $0-20$ & $14.4 \pm 3.8$ \\
\hline
\end{tabular}


Table A.5. Correlations between HEI-2010 and MVPA in Total Sample

\begin{tabular}{|l|c|c|}
\hline Category & Pearson Correlation $(\mathrm{r})$ & p-value \\
\hline Total HEI-2010 Score & 0.049 & 0.558 \\
\hline Total Vegetable & -0.055 & 0.507 \\
\hline Green and Bean & 0.102 & 0.217 \\
\hline Total Fruit & 0.061 & 0.461 \\
\hline Whole Fruit & 0.075 & 0.367 \\
\hline Whole Grain & 0.074 & 0.370 \\
\hline Total Dairy & -0.013 & 0.876 \\
\hline Total Protein & 0.125 & 0.130 \\
\hline Seafood and Plant & -0.031 & 0.706 \\
Proteins & & 0.174 \\
\hline Fatty Acid & 0.112 & 0.561 \\
\hline Sodium & -0.048 & 0.223 \\
\hline Refined Grains & -0.101 & 0.874 \\
\hline Discretionary Calories & 0.013 & \\
\hline
\end{tabular}

